\title{
Transient Suppression of Broadband Gamma Power in the Default-Mode Network Is Correlated with Task Complexity and Subject Performance
}

\author{
Tomas Ossandón, ${ }^{1,2}$ Karim Jerbi, ${ }^{1,2}$ Juan R. Vidal, ${ }^{1,2}$ Dimitri J. Bayle, ${ }^{1,2}$ Marie-Anne Henaff,,${ }^{1,2}$ Julien Jung, ${ }^{1,2}$ \\ Lorella Minotti, ${ }^{3,4}$ Olivier Bertrand, ${ }^{1,2}$ Philippe Kahane, ${ }^{3,4}$ and Jean-Philippe Lachaux ${ }^{1,2}$ \\ ${ }^{1}$ Lyon Neuroscience Research Center, INSERM U1028, CNRS UMR5292, Brain Dynamics and Cognition Team, F-69500 Lyon-Bron, France, ${ }^{2}$ Université \\ Claude Bernard Lyon 1, F-69000 Lyon, France, ${ }^{3}$ Neurology Department, Grenoble Hospital, F-38043 Grenoble, France, and ${ }^{4}$ INSERM U836, Grenoble \\ Institute of Neurosciences, F-38700 Grenoble, France
}

Task performance is associated with increased brain metabolism but also with prominent deactivation in specific brain structures known as the default-mode network (DMN). The role of DMN deactivation remains enigmatic in part because its electrophysiological correlates, temporal dynamics, and link to behavior are poorly understood. Using extensive depth electrode recordings in humans, we provide first electrophysiological evidence for a direct correlation between the dynamics of power decreases in the DMN and individual subject behavior. We found that all DMN areas displayed transient suppressions of broadband gamma $(60-140 \mathrm{~Hz})$ power during performance of a visual search task and, critically, we show for the first time that the millisecond range duration and extent of the transient gamma suppressions are correlated with task complexity and subject performance. In addition, trial-by-trial correlations revealed that spatially distributed gamma power increases and decreases formed distinct anticorrelated large-scale networks. Beyond unraveling the electrophysiological basis of DMN dynamics, our results suggest that, rather than indicating a mere switch to a global exteroceptive mode, DMN deactivation encodes the extent and efficiency of our engagement with the external world. Furthermore, our findings reveal a pivotal role for broadband gamma modulations in the interplay between task-positive and task-negative networks mediating efficient goal-directed behavior and facilitate our understanding of the relationship between electrophysiology and neuroimaging studies of intrinsic brain networks.

\section{Introduction}

Our ability to quickly perceive and react to events in the outside world depends on our level of vigilance. Our state of alertness and thus our performance can fluctuate over the course of a day or even over the time span of a few seconds. To date, the neural mechanisms that facilitate or hinder efficient task engagement are still poorly understood. Converging neuroimaging evidence supports the view that attention-demanding, goal-directed behavior is mediated not only by distributed patterns of cerebral activation but, remarkably, also by concurrent suppression of activity in a distinct set of brain areas (Shulman et al., 1997;

Received May 18, 2011; revised Aug. 11, 2011; accepted Aug. 16, 2011.

Author contributions: T.O., M.-A.H., and J.-P.L. designed research; T.O., K.J., J.R.V., D.J.B., L.M., P.K., and J.-P.L. performed research; T.O., K.J., J.R.V., D.J.B., J.J., O.B., P.K., and J.-P.L. analyzed data; T.O., K.J., and J.-P.L. wrote the paper.

Funding was provided by the Ministère de l'Education Nationale et la Recherche (France), the Fondation pour la Recherche Médicale, and the BrainSync FP7 European Project (Grant HEALTH-F2-2008-200728). We thank all patients for their participation, the staff of the Grenoble Neurological Hospital epilepsy unit, and Dominique Hoffmann, Patricia Boschetti, Carole Chatelard, Véronique Dorlin, and Martine Juillard for their support. We are grateful to Dr Bharat Biswal for helpful comments on a previous version of this manuscript.

The authors declare no competing financial interests.

Correspondence should be addressed to Dr. Karim Jerbi, INSERM U1028, Centre de Recherche en Neurosciences de Lyon, Equipe Dynamique Cérébrale et Cognition, Centre Hospitalier le Vinatier, Batiment 452, 95 Boulevard Pinel, F-69500 Lyon-Bron, France. E-mail: karim.jerbi@inserm.fr.

DOI:10.1523/JNEUROSCI.2483-11.2011

Copyright $\odot 2011$ the authors $\quad 0270-6474 / 11 / 3114521-10 \$ 15.00 / 0$
Corbetta and Shulman, 2002; Fox et al., 2005). Because of their intrinsically elevated activity during rest, these areas collectively form a network known as the default-mode network (DMN) (Raichle et al., 2001; Raichle and Mintun, 2006). While taskrelated activations often involve frontoparietal circuits such as the dorsal attention network, task-negative networks comprise medial and lateral core regions of the DMN, including posterior cingulate cortex, medial prefrontal cortex, lateral temporal cortex, and temporal parietal junction. Additionally, distinct deactivation patterns have also been reported in inferior parietal and ventrolateral prefrontal cortex areas and are thought to be key components of a ventral attention network (Corbetta et al., 2008). DMN deactivation has been associated with the transition from an interoceptive mode of self-directed cognition (Gusnard and Raichle, 2001; Mason et al., 2007) to an exteroceptive mode of outward attention (Raichle and Mintun, 2006; Raichle, 2009; Spreng et al., 2010). In addition, recent findings suggest that the DMN couples with the frontoparietal network in goal-directed cognition (Spreng et al., 2010). While the precise spatial characteristics of the DMN are amenable to imaging studies, its finegrained temporal properties and its relationship with behavior remain elusive. As a result, the precise role of DMN deactivations is not fully understood. In this study, we address this issue by monitoring neural population activity directly recorded in human default mode structures and, most importantly, by assessing 
its modulation as a function of task engagement and performance. Using extensive brain-wide, intracerebral depth recordings in surgical epilepsy patients performing a visual search task, we found that DMN neural populations display task-related, high-frequency power suppressions in the high gamma range $(60-140 \mathrm{~Hz})$. Above all, we reveal for the first time that the fine-scale temporal dynamics of such broadband gamma power suppression in DMN regions are tightly correlated with task demands and subject performance on a trial-by-trial basis. Our findings further suggest that efficient goal-directed behavior is mediated by an intricate interplay between anticorrelated networks of distributed broadband gamma power increases and decreases.

\section{Materials and Methods}

\section{Stimuli and experimental design}

The stimuli used in this study are an adaptation of a classical visual search test developed by Treisman and Gelade (1980). Each stimulus consisted of an array of 36 letters $(6 \times 6$ square array with 35 Ls and one T randomly arranged). Participants were instructed to search as fast as possible for the "T" and press a response button as soon as they found it. Two main experimental conditions were contrasted: an easy search condition and a difficult search condition (see Fig. $1 B$ ). In the EASY condition, the target was gray while all distracters were black. To dissociate correct from wrong responses the subjects were required to indicate whether the target was located in the upper or lower half of the display by pressing one of two response buttons. In the DIFFICULT condition, both distracters and target were gray. The difficult and easy condition stimuli were presented randomly for a fixed duration of $3 \mathrm{~s}$ and with an interstimulus interval of $1 \mathrm{~s}$. The stimuli were displayed on a 19 inch computer screen located 60 $\mathrm{cm}$ away from the subject. Each experiment consisted of 6 runs of $5 \mathrm{~min}$ recording blocks.

\section{Participants}

Intracranial recordings were obtained from 14 neurosurgical patients with intractable epilepsy (11 women, mean age: $32 \pm 10$ years) at the Epilepsy Department of the Grenoble Neurological Hospital (Grenoble, France). All patients were stereotactically implanted with multi-lead EEG depth electrodes. All electrode data exhibiting pathological waveforms were discarded from the present study. This was achieved in collaboration with the medical staff and was based on visual inspection of the recordings and by systematically excluding data from any electrode site that was a posteriori found to be located within the seizure onset zone. All participants provided written informed consent, and the experimental procedures were approved by the Institutional Review Board and by the National French Science Ethical Committee. Patient-specific clinical details are provided in Table 1.

\section{Electrode implantation}

Eleven to fifteen semi-rigid, multi-lead electrodes were stereotactically implanted in each patient. The stereotactic electroencephalography (SEEG) electrodes used have a diameter of $0.8 \mathrm{~mm}$ and, depending on the target structure, consist of 10 to 15 contact leads $2 \mathrm{~mm}$ wide and $1.5 \mathrm{~mm}$ apart (DIXI Medical Instruments). All electrode contacts were identified on each patient's individual stereotactic implantation scheme and were subsequently localized anatomically using Talairach and Tournoux's proportional atlas (Talairach and Tounoux, 1993). In addition, computer-assisted matching between a post-implantation CT scan and a preimplantation 3-D MRI dataset (VoXim R, IVS Solutions) allowed for direct visualization of the electrode contacts on the patients brain anatomy using ACTIVIS (developed by INSERM U1028, CERMEP, and UMR 5230). Whenever available, we also used a post-implantation MRI to verify electrode localization. Visual inspection of the electrodes superposed on each subject's individual MRI was used to check whether each SEEG electrode was located in gray or white matter.

\section{SEEG recordings}

Intracerebral recordings were conducted using a video-SEEG monitoring system (Micromed), which allowed the simultaneous data recording

\begin{tabular}{llll}
$\begin{array}{l}\text { Table 1. Participant description: age, gender, and description of epileptogenic } \\
\text { focus as determined by the clinical staff of Grenoble Neurological Hospital, } \\
\text { Grenoble, France }\end{array}$ & & \\
\hline Patient & Age & Gender & Epileptogenic zone \\
\hline S1 & 46 & M & Right temporal (anteromesial) \\
S2 & 42 & F & Left temporal (anterior) \\
S3 & 22 & F & Left frontal (operculum) \\
S4 & 21 & F & Right frontal (prefrontal) \\
S5 & 39 & F & Bi-frontal \\
S6 & 35 & F & Right temporoparietal \\
S7 & 45 & F & Right temporooccipital \\
S8 & 26 & F & Right frontal (premotor) \\
S9 & 37 & F & Right frontal (premotor) \\
S10 & 25 & F & Right central \\
S11 & 32 & F & Bi-temporal \\
S12 & 19 & F & Right temporal (posterobasal) \\
S13 & 17 & M & Left occipital \\
S14 & 47 & M & Left fronto (orbital)-insular \\
\hline
\end{tabular}

from 128 depth-EEG electrode sites. The data were bandpass filtered online from 0.1 to $200 \mathrm{~Hz}$ and sampled at $512 \mathrm{~Hz}$ in 12 patients (data from the other two patients were digitized at $1024 \mathrm{~Hz}$ ). At the time of acquisition the data are recorded using a reference electrode located in white matter, and each electrode trace is subsequently re-referenced with respect to its direct neighbor (bipolar derivations). This bipolar montage has a number of advantages over common referencing. It helps eliminate signal artifacts common to adjacent electrode contacts (such as the $50 \mathrm{~Hz}$ mains artifact or distant physiological artifacts) and achieves a high local specificity by cancelling out effects of distant sources that spread equally to both adjacent sites through volume conduction. The spatial resolution achieved by the bipolar SEEG is on the order of $3 \mathrm{~mm}$ (Lachaux et al., 2003; Kahane et al., 2006; Jerbi et al., 2009). Both spatial resolution and spatial sampling achieved with SEEG differ slightly from that obtained with subdural grid electrocorticography (Jerbi et al., 2009).

\section{Time-frequency analysis, gamma power, and envelope computations}

Task-induced modulations of power across time and frequency were obtained by standard time-frequency (TF) analysis using wavelets (Tallon-Baudry et al., 1997), and instantaneous signal amplitude profiles were computed using the Hilbert transform (Le Van Quyen et al., 2001). All wavelet-based TF map computations were performed with in-house software package for electrophysiological signal analysis (ELAN) developed at INSERM U1028, Lyon, France (Aguera et al., 2011). In addition to the use of wavelet-based analysis, we also examined the time course of frequency-specific signal envelope modulations using the Hilbert transform. Applying the Hilbert transform to the continuous recordings splits the data into instantaneous amplitude (i.e., envelope) and phase components in a given frequency interval. In addition to being a convenient measure of instantaneous amplitude modulations in a frequency range of interest, performing spectral analysis using the Hilbert transform was also used to confirm the TF results obtained independently by wavelet analysis. These comparisons provided an additional level of confidence in the results of spectral analysis reported throughout this study. For a comparison of wavelet decomposition and Hilbert transform, see Le Van Quyen et al. (2001). In the following, we describe the standard procedure used to compute task-related power modulations in the high gamma band $(60-140 \mathrm{~Hz})$. The same method was used for calculations in other frequency bands.

Step 1: estimating gamma-band envelope (i.e., time course of gammaband amplitude). Continuous SEEG signals were first bandpass filtered in multiple successive $10 \mathrm{~Hz}$ wide frequency bands (e.g., 8 bands from [60-70 Hz] to [130-140 Hz]) using a zero phase shift noncausal finite impulse filter with $0.5 \mathrm{~Hz}$ roll-off. Next, for each bandpass filtered signal we computed the envelope using standard Hilbert transform. The obtained envelope has a sampling rate of $64 \mathrm{~Hz}$ (i.e., one time sample every $15,625 \mathrm{~ms}$ ). Again, for each band this envelope signal (i.e., time-varying amplitude) was divided by its mean across the entire recording session 
and multiplied by 100 . This yields instantaneous envelope values expressed in percentage (\%) of the mean. Finally, the envelope signals computed for each consecutive frequency bands (e.g., 8 bands of $10 \mathrm{~Hz}$ intervals between 60 and $140 \mathrm{~Hz}$ ) were averaged together to provide one single time series (the high gamma-band envelope) across the entire session. By construction, the mean value of that time series across the recording session is equal to 100 . Note that computing the Hilbert envelopes in $10 \mathrm{~Hz}$ sub-bands and normalizing them individually before averaging over the broadband interval allows us to account for a bias toward the lower frequencies of the interval that would otherwise occur due to the $\sim 1 / f$ drop-off in amplitude.

Step 2: computing task-related time course of percentage gamma power modulations. As for the wavelet-based TF analysis, we also computed task-related "emergence" (i.e., stimulus-related or response-related increases or decreases in the amplitude of the gamma-band envelope). These modulations were computed by contrasting gamma-band amplitude during visual search to values obtained during the prestimulus baseline period ( -400 to $-100 \mathrm{~ms}$ ). To test for significant increases or decreases, we used a paired-sample Wilcoxon signed rank test followed by false discovery rate (FDR) correction across all time samples. The FDR approach yields a corrected threshold for significance (Genovese et al., 2002) (e.g., Fig. 3). Note that for visualization purposes, all power profiles are baseline corrected. Moreover, cluster-level gamma-band time courses were obtained by event-locked time domain averaging across trials and electrodes of the cluster (see Table 3 for cluster details).

\section{Mapping intracranial EEG data to standard MNI brain}

To obtain an anatomical representation of all significant power modulations for a given frequency band, we mapped pooled data from all subjects onto a standard Montreal Neurological Institute (MNI) brain. The value assigned to each node of the MNI brain represents the average of data from all recording sites located within a maximum distance of 15 $\mathrm{mm}$ from the node (for this purpose, all electrode coordinates were transformed from Talairach to common MNI space). The individual data averaged in this study represent $Z$ values (Wilcoxon test, FDR corrected) for each subject; thereby they represent statistically significant task-related increases or decreases of signal power in a given frequency band (compared to a baseline period, as described above). This data mapping procedure provides, above all, a convenient way to pool and visualize intracerebral EEG data from all subjects on a common MNI brain and is used to identify anatomo-functional regions of interest for more precise analysis at the individual level. Using statistical significance of gamma power suppression as functional criterion and its spatial distribution as anatomical criterion the following major anatomofunctional deactivation clusters were obtained: posterior cingulate cortex (PCC), medial prefrontal cortex (MPFC), left and right ventrolateral prefrontal cortex (VLPFC), temporal parietal junction (TPJ), lateral temporal cortex (LTC), and middle frontal gyrus (MFG). Table 3 lists the electrodes that define the gamma-band power increase and decrease clusters as well as the coordinates of these ROIs and their anatomical location (Brodmann areas). Most importantly, all statistical analysis provided throughout this study are based on single electrode or cluster-level statistical analysis using the estimated band-specific time series at each electrode of each subject (power envelopes obtained with Hilbert transform). In other words, the interpolated and averaged data represented on the MNI brain maps are used for visualization purposes and not for statistical analysis.

\section{Computing onset and duration of gamma-band power suppressions}

We used signed rank Wilcoxon tests, followed by FDR correction (Genovese et al., 2002), across time samples to assess the statistical significance $(p<0.05)$ of event-related increases or decreases in the time course of gamma-band power. Gamma-band power decrease (GBD), onset latency, and duration (see Table 2) were determined from gamma-power envelopes on the basis of statistical criteria (FDR; $p<0.05$ for the latencies and $t$ tests, $p<0.01$ for the cluster-level inference).

\section{Table 2. Mean duration of significant gamma power suppression in the two experimental conditions (easy and difficult search) computed for all electrodes of each cluster across all 14 participants}

\begin{tabular}{lllllc}
\hline & \multicolumn{4}{l}{ Duration of GBD (ms) } \\
\cline { 2 - 6 } & LTC & VLPFC & TPJ & PCC & MPFC \\
\hline Easy search (E) & $135( \pm 74)$ & $387( \pm 56)$ & $310( \pm 114)$ & $178( \pm 84)$ & $384( \pm 63)$ \\
Difficult search (D) & $470( \pm 72)$ & $689( \pm 115)$ & $383( \pm 149)$ & $560( \pm 117)$ & $1005( \pm 142)$ \\
$p$ value (Evs D) & 0.0072225 & 0.0054465 & 0.235342 & 0.006786 & 0.0003145 \\
\hline
\end{tabular}

The difference between GBD duration in easy and difficult search conditions is statistically significant (paired $t$ test, $p<0.0001$ ) for all clusters except the TPJ cluster.

\section{Evaluating the relationship between GBD and performance}

The link between GBD strength and speed of target detection was examined at two levels, cluster approach and single-trial analysis.

Cluster approach. For a given condition (e.g., difficult search), we sorted the trials into two groups: $50 \%$ fastest response trials (short reaction times) and 50\% slowest response trials (long reaction times). We computed mean GBD power separately for each group of trials using data from all electrodes of the power suppression cluster (task-negative cluster). Note that mean GBD was obtained by taking the mean of the amplitude over a fixed time window during visual search: [from 200 to 700 ] ms for stimulus-locked analysis and [from -900 to -200] for responselocked analysis. We tested for differences in GBD for the two groups of trials (50\% fastest vs 50\% slowest) using a paired $t$ test $(p<0.01)$.

Single-trial analysis. Using single-trial electrode data, we computed the correlation between instantaneous gamma power and the reaction times of the corresponding trials. This was performed for each time bin of a single trial power envelope, resulting in a time series of correlation coefficients. Scatter plots were then used to illustrate the relationship between reaction times and single-trial gamma power at $t_{\max }$, the time bin of peak correlation (e.g., Fig. 4D).

Gamma-band large-scale correlation and anticorrelation analysis We examined temporal correlations among gamma power envelopes from all recording sites located in task-positive networks (TPNs) and task-negative networks (TNNs) in each subject. TPN and TNN consist of electrode clusters with significant stimulus induced gamma-band power increases and decreases respectively (see Table 3 for anatomical details). For each pair of electrodes the correlation was computed as follows. For each electrode site a time series was obtained by concatenating multiple $1 \mathrm{~s}$ segments of gamma power envelope, one from each trial (from 0 to $1000 \mathrm{~ms}$ following stimulus onset). For instance, a dataset with 100 trials would yield a $100 \mathrm{~s}$ time series of raw gamma power envelope obtained during active task periods. Next, we calculated the cross-correlation between the two signals at various time lags (within $\pm 156 \mathrm{~ms}$ range) to identify the maximum correlation value (i.e., maximum in terms of absolute value). Next, we computed correlations obtained with surrogate datasets obtained by recomposing one of the two signals via random shuffling of the trial order within the time series. This was carried out 99 times for each electrode pair, yielding 99 correlation values. If the original correlation between the two time series is higher (in absolute value) than the 99 surrogates, the correlation between the gamma envelopes at the two sites was considered to be statistically significant at $p<0.01$. After computing all possible correlations between all relevant electrode pairs within each subject, we evaluated the number of significant correlations as a function of the type of electrode pair group to which they belong: intranetwork correlation (i.e., TPN-TPN and TNA-TNA electrode pairs) or internetwork correlation (i.e., TPN-TNA electrode pairs). The total number of significant positive and negative correlations and their ratio with respect to nonsignificant correlation was computed as a function of correlation type (internetwork or intranetwork correlation). Finally, we used paired $t$ test across subjects to test whether the negative or rather the positive correlations were predominant in each correlation group (internetwork correlations and intranetwork correlations). 
Table 3. Anatomical details of the task positive network and the task negative network

\begin{tabular}{|c|c|c|c|c|c|c|}
\hline & Cluster & Brodmann area(s) & No. of electrode pairs & No. of patients (of 14) & ROI center (right hemisphere) & ROI center (left hemisphere) \\
\hline TNN & PCC & 31 & 11 & 5 & $(-4,-53,30)$ & $(-4,-53,30)$ \\
\hline TNN & VLPFC & $45 / 47$ & 26 & 6 & $(50,34,6)$ & $(-49,37,6)$ \\
\hline TNN & MPFC & $32 / 10$ & 12 & 5 & $(10,45,4)$ & $(10,45,4)$ \\
\hline TNN & TPJ & 40 & 7 & 4 & $(53,-31,43)$ & $(-57,27,52)$ \\
\hline TNN & LTC & 21 & 12 & 5 & $(61,-31,-7)$ & $(-61,-34,-4)$ \\
\hline TNN & MFG & 9 & 6 & 3 & $(43,8,40)$ & $(-39,47,25)$ \\
\hline TPN & DLPFC & 46 & 12 & 4 & $(43,34,26)$ & $(-50,11,16)$ \\
\hline TPN & IPS & 7 & 5 & 2 & $(23,-50,49)$ & n.a. \\
\hline TPN & IPL & $7 / 40$ & 8 & 3 & $(40,-41,41)$ & n.a. \\
\hline TPN & FEF & 6 & 12 & 3 & $(12,-10,56)$ & $(-18,-5,49)$ \\
\hline TPN & SMA/Pre-SMA & 6 & 6 & 3 & $(6,-3,54)$ & $(-10,4,49)$ \\
\hline TPN & Inf prec gyrus & $6 / 32$ & 35 & 3 & $(38,-6,56)$ & $(-38,-4,40)$ \\
\hline TPN & MT & $19 / 37$ & 51 & 7 & $(45,-61,0)$ & $(-42,-58,-3)$ \\
\hline TPN & Orbital gyrus & $19 / 18 / 23$ & 22 & 4 & $(37,-76,11)$ & $(-24,-72,0)$ \\
\hline
\end{tabular}

Electrodes that show statistically significant decrease or increase in gamma-band power (compared to prestimulus baseline levels) were assigned to the TNN or TPN group respectively (compare Fig. 6). Electrodes within the TNN and TPN were grouped into multiple anatomical clusters. Given the combination of functional (i.e. statistically significant gamma power modulation, Wilcoxon test, $p<0.05$, FDR correction) and anatomical criteria, the individual clusters within TNN and TPN represent anatomofunctional regions of interest (ROIs). The center of each cluster is obtained by computing the centroid of the Talairach coordinates of all recording sites it includes. Note that the coordinates of PCC, MPFC, and TPJ ROIs are consistent with the coordinates of these DMN structures as reported in the fMRI literature (e.g., Fox et al., 2005). DLPFC, Dorsolateral prefrontal cortex; FEF, frontal eye field; Inf prec gyrus, inferior precentral gyrus; IPL, inferior parietal lobule; IPS intraparietal sulcus; MT, middle temporal cortex; SMA, supplementary motor area.

\section{Results}

Transient gamma power suppressions in $\mathrm{DMN}$ areas during task performance We obtained a spatially dense sampling of the human brain by acquiring SEEG data from 1730 intracerebral recording sites in 14 patients who underwent neurosurgical treatment for epilepsy (Fig. 1A). Participants were presented with a search array and were instructed to detect a target (a T among the Ls) as fast as possible. We computed task-related spectral power modulations across time and frequency at all recording sites in two experimental conditions: (1) an easy search; and (2) a difficult search (Fig. 1B). Remarkably, during visual search a subset of recording sites displayed strong power suppressions in the high-gamma range $(60-140 \mathrm{~Hz})$ that was occasionally associated with power increases at lower $(<30 \mathrm{~Hz})$ frequencies, as illustrated in Figure $1 C$. To evaluate the spatial distribution of such broadband gamma power suppressions during visual search across the entire brain, we mapped all statistically significant task-related gamma-band decreases, GBDs, from all individuals onto a normalized common brain (see Materials and Methods). The results in Figure $2 \mathrm{~A}$ show that the anatomical distribution of GBD bears a striking similarity with the classical DMN areas (Raichle et al., 2001). In addition to PCC, MPFC, and TPJ, we also observed statistically significant suppressions bilaterally in VLPFC, LTC, and in right MFG.

\section{Correlation between subject behavior and gamma power suppression}

Analysis of the behavioral data showed that correct target detections (achieved within the fixed $3 \mathrm{~s}$ time window) dropped from $91 \%$ hits in the easy search to $77 \%$ hits in the difficult search. Mean subject reaction times were significantly faster in the easy
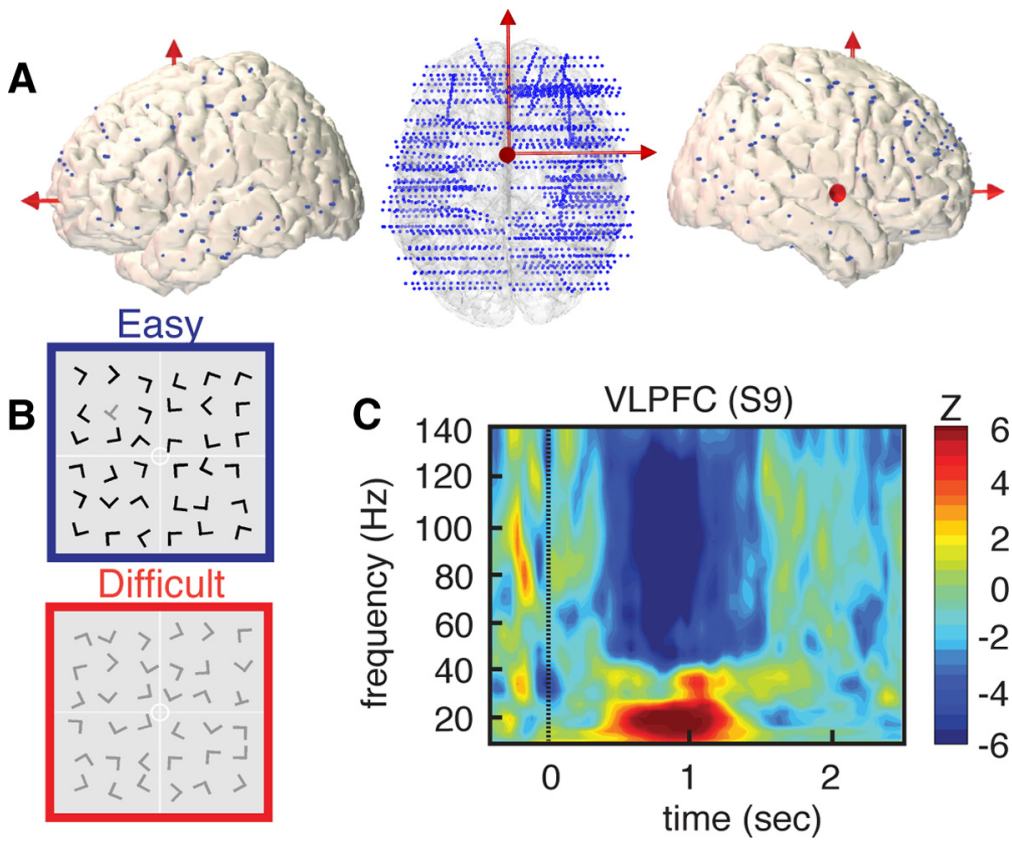

Figure 1. High-density depth electrode recordings during visual search. $\boldsymbol{A}$, Left, Top and right side views of implanted electrode locations represented on a 3-D reconstruction of a standard (MNI) brain. Blue dots represent the Talairach coordinates for all contacts on all SEEG electrodes for all 14 subjects (total $=1730$ recording sites). $\boldsymbol{B}$, Example of visual search arrays for easy (blue) and difficult (red) conditions (subjects were asked to find the T among the Ls). C, Strong power suppression in broad-band gamma $(60-140 \mathrm{~Hz})$ in right VLPFC during visual search. High gamma suppression also coincides with power increases in lower $(<30 \mathrm{~Hz})$ frequency range. The illustrative time-frequency map represent increases and decreases in spectral power compared to a prestimulus baseline level (baseline, [from -400 to -100 ] ms, Wilcoxon Z value).

search $(1009 \pm 59 \mathrm{~ms})$ than the difficult search $(1678 \pm 79 \mathrm{~ms})$ conditions $(t=34.24, p<0.0001)$, suggesting longer task engagement during the difficult search. Remarkably, the direct electrophysiological recordings in all the GBD clusters reveal a longer lasting suppression of gamma power in the difficult search condition compared to the easy search condition (see Fig. $2 B, D$ for cluster results and Fig. 3 for individual electrode data). In other words, the gamma suppression phenomenon lasted longer with higher task complexity and longer task engagement. Furthermore, mean onset times of significant gamma suppression in each cluster were found to indicate that the earliest power de- 
A
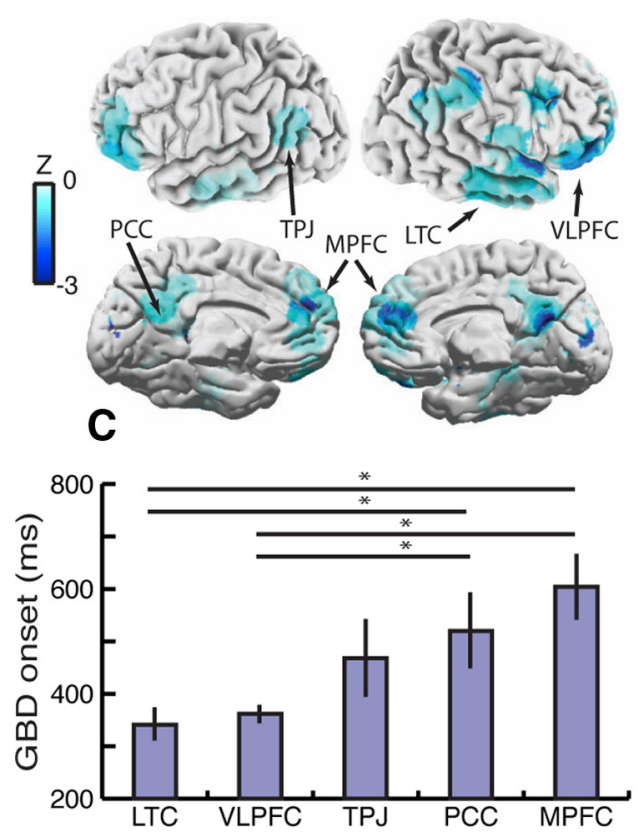

B $\quad$ EEasy $\square$ Difficult
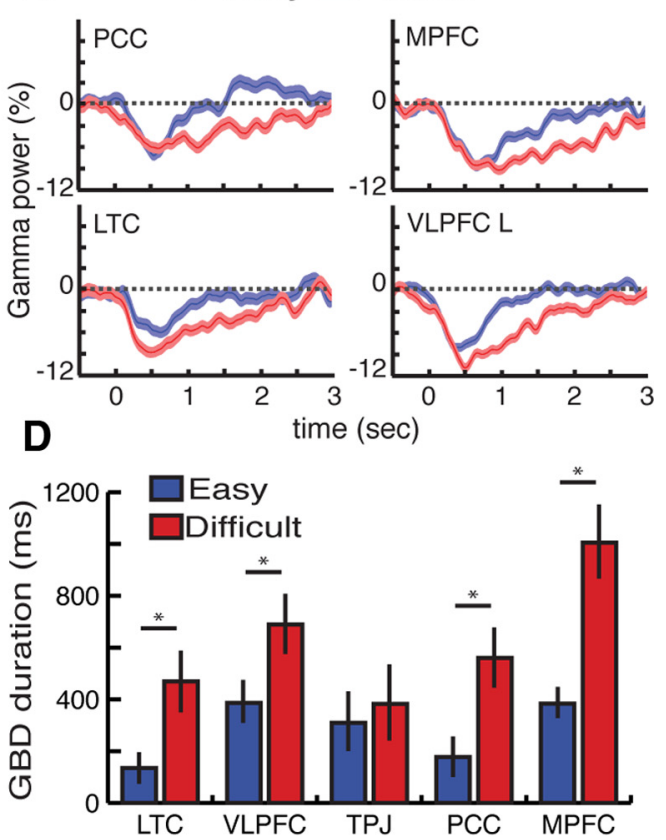

Figure 2. Brain-wide dynamics of gamma-band power decrease, GBD, during visual search. $\boldsymbol{A}$, Anatomical distribution of statistically significant broad-band gamma (60 - $140 \mathrm{~Hz}$ ) suppression obtained by mapping depth electrode data in all subjects $(n=14)$ to a standard brain. The spatial properties of GBD bear a striking resemblance to DMN maps previously reported with fMRI. Data snapshot at $t=640 \mathrm{~ms}$ following search array presentation (only power decreases are shown here; see Fig. 5 for power increases and decreases and Materials and Methods for details) $\boldsymbol{B}$, GBD temporal profile during easy (blue) and difficult (red) search for four illustrative clusters: PCC, MPFC, LTC, and VLPFC left (L). Time $t=0$ ms indicates onset of search array display. C, Mean GBD onset for each cluster. Values represent mean onset latency, i.e., time sample at which deactivation reached statistical significance (group effect $F_{(4,98)}=6.817, p<0.0001 ;$ Tukey's HSD Test $\left(^{*}\right)$ indicates $p<0.05)$. D, Duration of GBD for easy (blue) and difficult (red) visual search conditions. Values represent mean duration of significant deactivation across all electrodes of each cluster ( $t$ test $(*)$ indicates $t>2.76, p<0.01$ ). See Table 2 for the details of task-related GBD duration and associated $p$ values and Table 3 for GBD cluster details.

creases occurred in LTC and VLPFC clusters, followed by TPJ, PCC, and eventually MPFC [Fig. $2 C$, group effect $F_{(4,98)}=6.817$, $p<0.0001$; Tukey's honestly significant difference (HSD) test, $p<0.05]$. Interestingly, the chronology of gamma power suppression in these clusters was the same regardless of task difficulty. Next, we addressed the link between the task-related gamma power decrease and behavioral performance by assessing the putative relationship between the strength of gamma suppression and target detection speed. First, when splitting the data recorded in all anatomical clusters into two subgroups of trials, $50 \%$ shortest versus $50 \%$ longest reaction times, we found quicker target detection to be associated with stronger gamma suppression in MPFC and VLPFC clusters (Fig. 4A). The correlation between GBD amplitude and performance (target detection speed) was thus not as ubiquitous across the task-negative network as the correlation between GBD duration and task complexity. Furthermore, the relationship between power suppression and reaction time was also investigated in individual electrode data (e.g., Fig. $4 B-E$ ). Remarkably, single-trial power analysis in individual electrodes revealed statistically significant correlation between gamma suppression and target detection speed on a trial-by-trial basis (cf. illustrative example in Fig. 2C-E, correlation peak rho $=0.34968$ at $t=736 \mathrm{~ms}, p<0.001$, Spearman's correlation test).

\section{Functional specificity of broadband gamma power suppression}

Furthermore, we also computed brain-wide, task-related power modulations across theta $(4-7 \mathrm{~Hz})$, alpha $(8-12 \mathrm{~Hz})$, beta $(13-30$ $\mathrm{Hz})$ and low gamma $(30-60 \mathrm{~Hz})$ bands. Compared to lower frequencies, task-related (low and high) gamma suppression pat- terns displayed the closest anatomical match with typical DMN deactivation networks (Fig. 5). Note that power suppressions were also observed in the beta-band in some DMN areas (e.g., PCC). However, by contrast to GBD, beta-band decreases were neither correlated with task complexity nor with subject performance (correlations did not achieve statistical significance, $p>$ $0.05)$. Furthermore, beta-band power suppression was not restricted to DMN structures (see Fig. 5). Modulations of low-gamma (30-60 $\mathrm{Hz}$ ) power suppression showed statistically longer lasting decreases in MPFC in the difficult compared to the easy search condition as well as stronger reductions in LTC in association with faster responses ( $p<0.01$, $t$ test, both $t>2.73$ ) (data not shown). Nevertheless, low-gamma modulations failed to achieve the level of significance and consistency across the full DMN network that was observed in the high gamma range $(60-140 \mathrm{~Hz})$.

\section{Relationship between task-positive and task-negative gamma modulation networks}

In addition to identifying the dynamics of GBD and its relationship with behavior, we also investigated task-related gamma band activation (GBA) and the putative interplay between task-related negative and positive gamma power modulations during visual search. Figure $6 \mathrm{~A}$ depicts the brain-wide spatial distribution of task-related GBD and GBA forming the task-negative network and the task-positive network, respectively. Furthermore, we computed exhaustive pairwise correlation analysis between the time courses of gamma power modulation across both networks. The global correlation results (Fig. 6B,C) indicate that TPN and TNN are predominantly negatively correlated (i.e., anticorrelated), whereas TNN-TNN and TPN-TPN pairs of sites are predominantly positively correlated (see Materials and Methods for 
details). This result matches patterns of correlated and anticorrelated networks of intrinsic brain organization (Fox et al., 2005) revealed using resting-state functional magnetic resonance imaging (fMRI) with the notable difference, however, that here it was obtained during active task performance and by measuring high gammaband power modulations.

\section{Discussion}

This study provides the first direct evidence for a correlation between behavioral parameters and task-related gamma-band suppression dynamics in DMN. Our findings indicate that complex tasks are associated with longer lasting power decreases than easier tasks, and that stronger gamma suppressions in distinct DMN components are correlated with better performances (i.e., faster target detection).

Studies in animals (Logothetis et al., 2001; Niessing et al., 2005) and humans (Mukamel et al., 2005; Lachaux et al., 2007; Nir et al., 2007; He et al., 2008) suggest that increases in hemodynamic responses are not only linked to increases in spiking activity but also to power increases in the gamma-band component of local field potentials (LFPs). Conversely, here we demonstrate, using direct electrophysiological recordings in the human brain, that all DMN areas, which are known to exhibit negative BOLD responses, display suppressions of gammaband power during task engagement. Critically, our results significantly extend previous invasive studies of task-related gamma power suppressions in humans (Lachaux et al., 2008; Miller et al., 2009; Jerbi et al., 2010; Dastjerdi et al., 2011) and monkeys (Shmuel et al., 2006; Hayden et al., 2009). In addition to probing DMN electrophysiology via an unprecedented large-scale sampling of the human brain (14 subjects, 1730 recording sites), the present study is above all the first to reveal the millisecond temporal features (onset and duration) of cortical gamma power decreases across the entire default-mode network, and it is also the first to report significant correlations between the transient power suppressions and behavior (duration of task engagement and performance). The highly transient nature of the DMN power suppressions reported here and the sequential order of the power drop onset in each of the various nodes of the network highlight the dynamic nature of the network, a feature that is likely to facilitate rapid switching between different attentional states or degrees of engagement with the external world.

A significant side result from this study is that it provides additional irrefutable electrophysiological evidence for a neural origin of task-related DMN deactivation in agreement with recent studies (Hayden et al., 2009; Miller et al., 2009; Jerbi et al., 2010). This firmly settles the recurrent controversy sparked by

\section{Easy $\square$ Difficult}

$\operatorname{VLPFC~L~(S4,~gp14)~}$
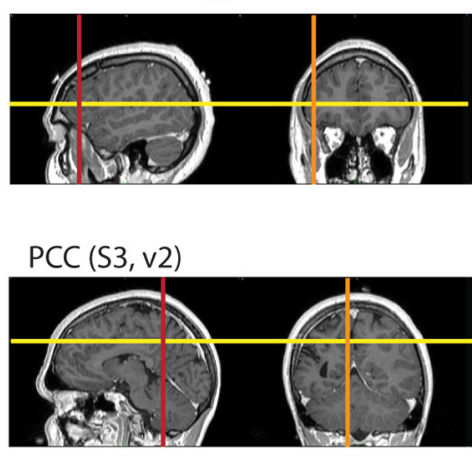

$\operatorname{VLPFC} R(S 9, g 15)$

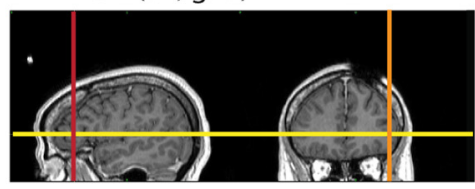

$\operatorname{MPFC}(\mathrm{S9}, \mathrm{gp} 4)$

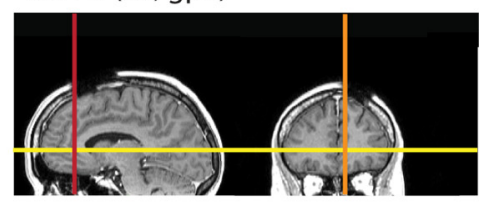

LTC (S13, cp11)

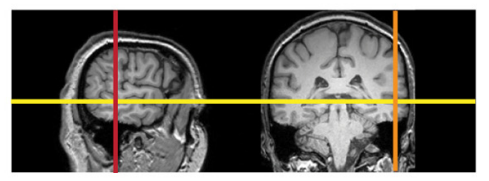

0 time (sec)

Figure 3. Single electrode data show suppression of gamma-band activity varies with task difficulty. Temporal profile of gamma band $(60-140 \mathrm{~Hz})$ power modulations are displayed for the two conditions: easy search (blue) and difficult search (red) for representative sites in the task-negative (i.e., power suppression) network. The displayed data correspond to electrodes located in (from top to bottom): VLPFC left (L), PCC, VLPFC right (R), MPFC, and LTC. Horizontal red and blue lines, beneath the waveforms, rank Wilcoxon test and FDR correction $(p<0.05)$. Line upper/lower bounds indicate \pm 1 SEM. Note that the anatomical ROI name above each MRI panel is followed by subject number and electrode name.

suggestions that deactivations in DMN may arise from nonneuronal signals such as cardiac or respiratory artifacts (Birn et al., 2008) and vascular or metabolic changes (Pasley et al., 2007). Most importantly, our data argue against this claim not only because power suppressions were recorded directly in DMN structures, but also because the duration and amplitude of the power suppressions were correlated with the attentional demands of the task and with subject performance.

Compared to previous noninvasive studies based on scalp electroencephalography or magnetoencephalography (MEG) (Laufs et al., 2003; Mantini et al., 2007; Scheeringa et al., 2008; de Pasquale et al. 2010; Brookes et al., 2011), the direct intracranial recordings used here allow for a higher spatial resolution particularly for deeper structures, and for the detection of electrophysiological correlates of the DMN at higher frequencies (up to 200 

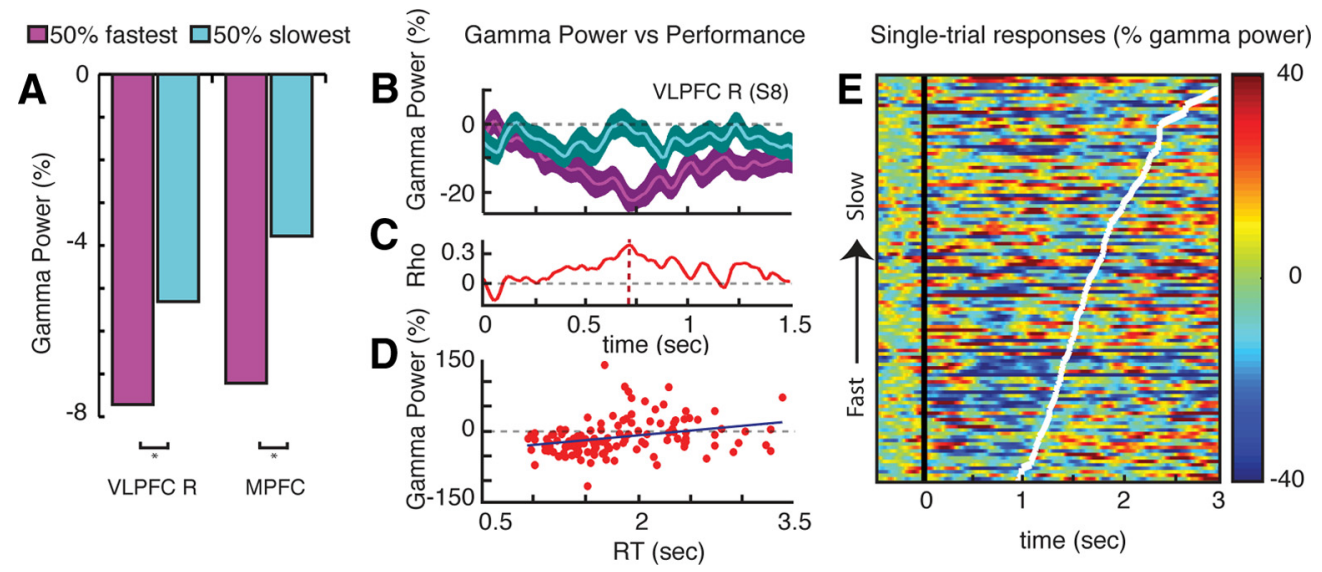

Figure 4. Relationship between gamma-band power decreases and behavioral performance. A, Mean gamma power suppression computed separately for $50 \%$ fastest response trials (magenta) and 50\% slowest response trials (turquoise). Gamma power suppressions in MPFC and right VLPFC (VLPC R) clusters were stronger for fast target detection than for the slower detections (both $t>$ $3.11, p<0.01$ ). Differences were not statistically significant for other GBD clusters (data not shown). Mean power was computed over a [200-700] ms time window following stimulus presentation. $B$, Profile of mean gamma power ( \pm SE) in a VLPFC R recording site (Talairach coordinates: $x=55, y=33, z=0$ ) for $50 \%$ fastest (magenta) versus $50 \%$ slowest (turquoise) response trials. $C$, Profile of correlation coefficient between single-trial gamma power at this site and the individual reaction times. A peak correlation occurred at $t_{\max }=736 \mathrm{~ms}$ after stimulus onset. $\boldsymbol{D}$, Trial-by-trial plot of reaction time (RT) versus gamma power at $t_{\text {max }}$ depicts the significant correlation between single-trial gamma suppression and behavioral performance $($ Spearman's correlation test, rho $=0.34968$, $p=0.0000969)$. $\boldsymbol{E}$, Single-trial gamma power plot for same electrode as in $\boldsymbol{B}-\boldsymbol{D}$ with trials sorted according to RTs (fastest to slowest target detection). White line depicts RT (i.e., latency of button press indicating target detection for each trial. Time $t=0$ corresponds to visual search array onset.

A

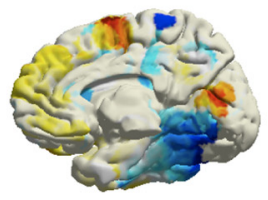

B

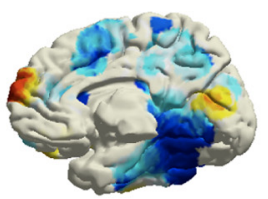

C
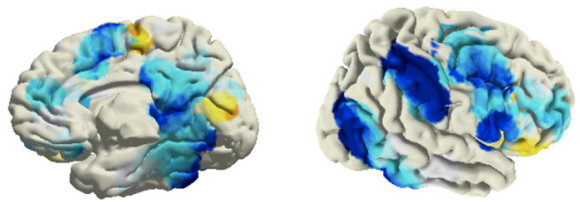

Beta $(15-30 \mathrm{~Hz})$
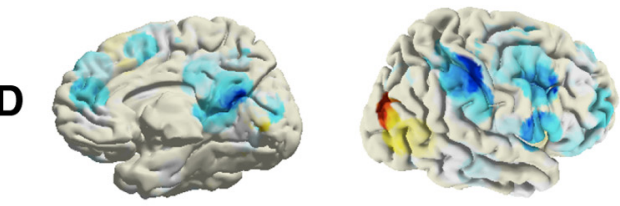

Low gamma $(30-60 \mathrm{~Hz})$

E
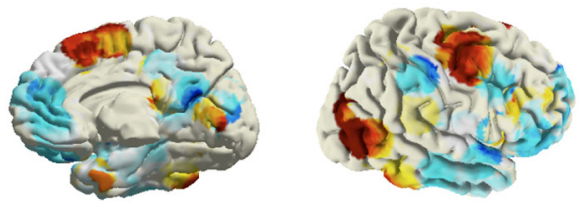

High gamma $(60-140 \mathrm{~Hz})$

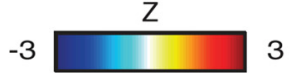

Figure 5. Spatial distribution of task-related power decreases (blue) and increases (red) during visual search in multiple frequency bands. $\boldsymbol{A}$, Theta $(4-7 \mathrm{~Hz})$. B, Alpha band $(8-12 \mathrm{~Hz})$. C, Beta band (13-30 Hz). D, Low gamma band $(30-60 \mathrm{~Hz})$. $\boldsymbol{E}$, High gamma band $(60-140 \mathrm{~Hz})$. The distribution of task-related gamma power suppressions is the one that most closely matches typical DMN deactivation patterns. Power patterns in theta and alpha bands did not show comparable suppressions in DMN areas. Beta power suppressions were observed in some DMN areas but also in visual and dorsal attention network areas. Unlike gamma suppressions, beta suppressions in the DMN clusters were not significantly correlated with behavior (data not shown).

$\mathrm{Hz})$. Incidentally, these two properties are critical for an optimal investigation of the electrophysiological correlates of DMN (Jerbi et al., 2010). This suggests that intracranial EEG studies might be essential for the reliable study of DMN electrophysiology in humans. Nevertheless, it is important to keep in mind that one major asset of scalp EEG is that it can be acquired simultaneously with fMRI (Laufs et al., 2003; Mantini et al., 2007; Scheeringa et al., 2008; de Pasquale et al. 2010;). We therefore expect fMRI invasive and noninvasive EEG approaches to play increasingly complementary roles to elucidate the neural underpinnings of intrinsic brain networks and to bridge the gap between imaging studies and electrophysiology.

Although the precise role of resting state networks is not yet fully understood, recent fMRI investigations suggest that DMN deactivation is correlated with task demands and behavioral parameters (Weissman et al., 2006; Singh and Fawcett, 2008). Our ability to directly measure task-related neural population activity with electrodes implanted in DMN structures significantly expands on previous investigations of the functional role of DMN. First of all, by contrast to the hemodynamic response that represents an indirect measure of neural activity, the brain-wide, high-resolution depth electrode recordings reported here allow for a direct assessment of the electrophysiological basis of the DMN phenomenon in humans. Second, the high signal-to-noise ratio of intracerebral recordings allowed 

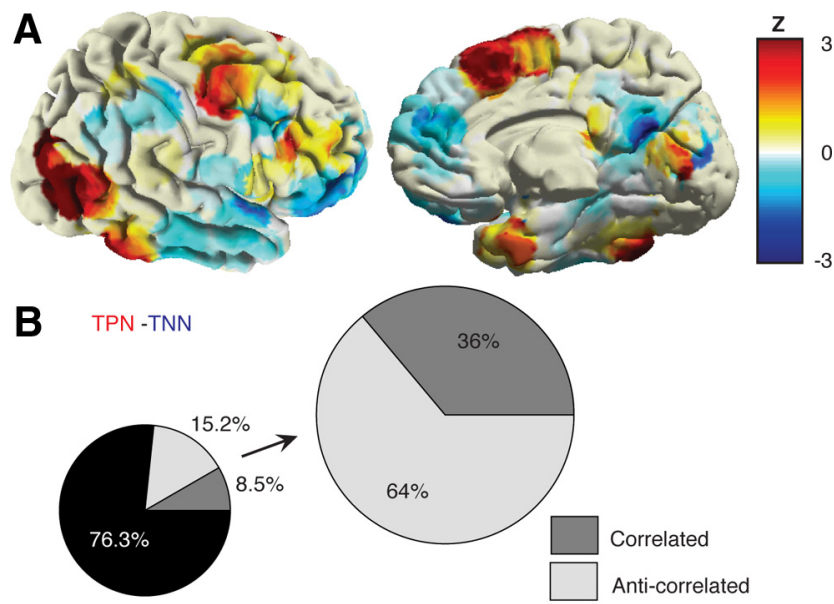

C

TPN -TPN TNN - TNN
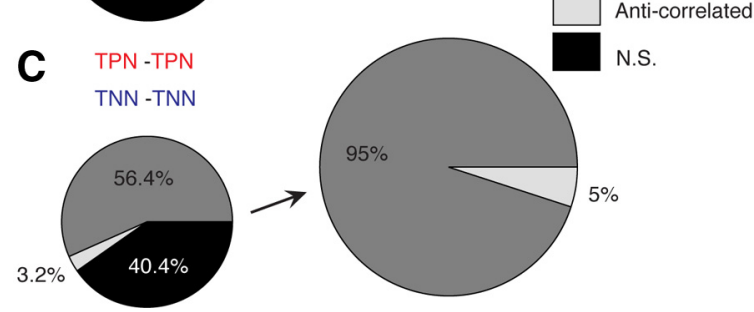

Figure 6. Correlated and anticorrelated gamma-band task networks. A, Spatial distribution of task-related gamma decreases (blue) and increases (red) during visual search, representing a task negative network and a task positive network, respectively. While TNN closely matches DMN and ventral attention network areas, TPN depicts concurrent activations in visual and dorsal attention network areas (data represent a snapshot of gamma power modulation at $t=$ $640 \mathrm{~ms}$ as compared to baseline level). $\boldsymbol{B}$, Anticorrelated gamma networks. These measure correlation among TPN-TNN by assessing correlation between single-trial data from all electrodes of TPN ROIs and TNN ROIs. $\boldsymbol{C}$, Correlated gamma networks. These are the same as in $\boldsymbol{B}$, but electrode pairs for the correlation analysis were always from the same type of network i.e., TPN-TPN and TNN-TNN correlations (TPN and TNN anatomical regions and electrode details are provided in Table 3).

us to perform trial-by trial data analysis revealing, for the first time, a significant correlation between an electrophysiological feature of DMN dynamics and subject performance on a trial-bytrial basis. Third, our study unravels the profile of DMN power suppression with a temporal resolution, allowing us to precisely determine the onset times and duration of the power decrease across the full DMN network. Remarkably, this fine-grained evaluation of DMN temporal dynamics as a function of task complexity unraveled the strikingly transient nature of the reduction in DMN gamma power. In fact, the high temporal resolution of intracerebral EEG may be key to fine-tuning the functional dissociation between the DMN and the ventral frontoparietal network structures in goal-directed behavior (Corbetta et al., 2008; Spreng et al., 2010). As a matter of fact, our finding that task engagement induced neural power suppression first in LTC, VLPFC, and TPJ structures before it occurred in PCC and MPFC. This observation could be taken as an indication that the ventral attention network is deactivated before the canonical DMN structures in this visual search task. Whether this fine temporal dissociation between the two networks holds true for other attention-demanding tasks is an exciting question for future intracranial EEG studies.

Moreover, accumulating evidence supports a direct relationship between broadband gamma and spiking activity (Nir et al., 2007; Ray et al., 2008; Manning et al., 2009; Ray and Maunsell, 2011). Remarkably, electrophysiological recordings in the macaque have shown that gamma-band suppression in LFP signals is associated with a decrease in neuronal firing (Shmuel et al.,
2006; Hayden et al., 2009). Taken in conjunction with these reports, our results suggest that DMN deactivation in humans is also likely to coincide with transient task-related drops in neuronal firing. Beyond uncovering a key role for broadband gamma activity in the electrophysiological mechanisms underlying DMN power suppression, our results have direct implications on the functional interpretation of task-related neural deactivation. The correlation we found between the decrease in DMN power and subject behavior suggests that beyond indicating a switch from an inattentive or introspective state to an attentive exteroceptive mode, the extent and duration of DMN suppression actually encode the quality of this transition, i.e., the efficiency and extent of our engagement with the external world. Critically, the remarkably transient nature of broadband gamma suppression in the default mode and ventral attention networks indicates that taskrelated reduction in cortical power is followed by a rapid return to baseline levels immediately after completion of task processing. As shown in Table 2, the mean duration of task-related gamma suppression was as short as $135 \mathrm{~ms}$ in LTC (easy search) and could last up to $1005 \mathrm{~ms}$ in MPFC (difficult search).

Additionally, our results point toward a prominent role for the network interplay between task-specific gamma increases and task-unspecific gamma decreases in mediating goal-directed behavior. Incorporating these findings in future research will further narrow the gap between fMRI studies and electrophysiology and will advance our knowledge of large-scale neural synchronization in the gamma band (Varela et al., 2001; Lakatos et al. 2008; Fries, 2009; Luo et al., 2010). In particular, our findings suggest that modulations of broadband gamma activity can be observed locally by task-related enhancement or suppression of power, but also through task-related, long-range, amplitude-amplitude correlations and anticorrelations in the same frequency range. Our understanding of the functional architecture of the human brain is being reshaped by resting-state fMRI studies that show how multiple brain areas form distinct, large-scale, coherent networks (Biswal et al., 1995, 2010). Investigating the electrophysiological correlates of spontaneous coherent fluctuations with the techniques used in this study will provide critical insights into the neural mechanisms mediating fMRI functional connectivity. In particular, applying coupling measures to direct electrophysiological recordings obtained simultaneously from multiple DMN nodes will be critical to unravel the frequency characteristics and directionality of large-scale slow correlated oscillations and their putative relationship with broadband gamma amplitude fluctuations (Jensen and Colgin, 2007; Lakatos et al. 2008; Dalal et al., 2011).

Because we did not have the possibility to acquire functional MRI data from the patients we examined here, we cannot completely rule out that the properties of the DMN in some of these subjects might have been directly or indirectly affected by epilepsy. Indeed, previous studies have reported possible alterations of the DMN in some epilepsy patients (e.g., Broyd et al., 2009, Laufs et al., 2007; Sakurai et al., 2010, Zhang et al., 2010). Future studies that combine fMRI and intracranial EEG exploration of the DMN in the same population will be key to distinguishing the physiological and pathological aspects of the electrophysiological underpinnings of the DMN. In the present study, we minimize the effect of epilepsy-related activity by excluding data from any electrode site that showed epileptic discharges or that was identified as being part of the seizure onset zone.

Finally, our data suggest that broadband gamma modulations could prove to be an efficient electrophysiological biomarker, complementing resting-state fMRI approaches, to investigate the 
pathophysiology of neurological diseases that involve DMN dysfunction. These include attention deficit hyperactivity disorders, autism, depression, Alzheimer's disease, and schizophrenia (Greicius et al., 2004; Garrity et al., 2007; Castellanos et al., 2008; Broyd et al., 2009; Uhlhaas and Singer, 2010). For such a perspective to become clinical routine, the challenge remains to localize broadband gamma brain signals using noninvasive methods (e.g., MEG or EEG) with sufficiently high spatial and temporal resolution (Jerbi et al., 2009) to warrant the type of DMN assessment achieved here with intracerebral recordings.

\section{References}

Aguera PE, Jerbi K, Caclin A, Bertrand O (2011) ELAN: a software package for analysis and visualization of MEG, EEG, and LFP signals. Comput Intell Neurosci 2011:158970.

Birn RM, Murphy K, Bandettini PA (2008) The effect of respiration variations on independent component analysis results of resting state functional connectivity. Hum Brain Mapp 29:740-750.

Biswal BB, Mennes M, Zuo XN, Gohel S, Kelly C, Smith SM, Beckmann CF, Adelstein JS, Buckner RL, Colcombe S, Dogonowski AM, Ernst M, Fair D, Hampson M, Hoptman MJ, Hyde JS, Kiviniemi VJ, Kötter R, Li SJ, Lin CP, et al. (2010) Toward discovery science of human brain function. Proc Natl Acad Sci U S A 107:4734-4739.

Biswal B, Yetkin FZ, Haughton VM, Hyde JS (1995) Functional connectivity in the motor cortex of resting human brain using echo-planar MRI. Magn Reson Med 34:537-541.

Brookes MJ, Wood JR, Stevenson CM, Zumer JM, White TP, Liddle PF, Morris PG (2011) Changes in brain network activity during working memory tasks: a magnetoencephalography study. Neuroimage 55:1804-1815.

Broyd SJ, Demanuele C, Debener S, Helps SK, James CJ, Sonuga-Barke EJ (2009) Default-mode brain dysfunction in mental disorders: a systematic review. Neurosci Biobehav Rev 33:279-296.

Castellanos FX, Margulies DS, Kelly C, Uddin LQ, Ghaffari M, Kirsch A, Shaw D, Shehzad Z, Di Martino A, Biswal B, Sonuga-Barke EJ, Rotrosen J, Adler LA, Milham MP (2008) Cingulate-precuneus interactions: A new locus of dysfunction in adult attention-deficit/hyperactivity disorder. Biol Psychiatry 63:332-337.

Corbetta M, Shulman GL (2002) Control of goal-directed and stimulusdriven attention in the brain. Nat Rev Neurosci 3:201-215.

Corbetta M, Patel G, Shulman GL (2008) The reorienting system of the human brain: From environment to theory of mind. Neuron 58:306 -324.

Dalal SS, Vidal JR, Hamamé CM, Ossandón T, Bertrand O, Lachaux JP, Jerbi K (2011) Spanning the rich spectrum of the human brain: slow waves to gamma and beyond. Brain Struct Funct 216:77-84.

Dastjerdi M, Foster BL, Nasrullah S, Rauschecker AM, Dougherty RF, Townsend JD, Chang C, Greicius MD, Menon V, Kennedy DP, Parvizi J (2011) Differential electrophysiological response during rest, selfreferential, and non-self-referential tasks in human posteromedial cortex. Proc Natl Acad Sci U S A 108:3023-3028.

de Pasquale F, Della Penna S, Snyder AZ, Lewis C, Mantini D, Marzetti L, Belardinelli P, Ciancetta L, Pizzella V, Romani GL, Corbetta M (2010) Temporal dynamics of spontaneous MEG activity in brain networks. Proc Natl Acad Sci U S A 107:6040-6045.

Fox MD, Snyder AZ, Vincent JL, Corbetta M, Van Essen DC, Raichle ME (2005) The human brain is intrinsically organized into dynamic, anticorrelated functional networks. Proc Natl Acad Sci U S A 102:9673-9678.

Fries P (2009) Neuronal gamma-band synchronization as a fundamental process in cortical computation. Annu Rev Neurosci 32:209-224.

Garrity AG, Pearlson GD, McKiernan K, Lloyd D, Kiehl KA, Calhoun VD (2007) Aberrant "default mode" functional connectivity in schizophrenia. Am J Psychiat 164:450-457.

Genovese CR, Lazar NA, Nichols T (2002) Thresholding of statistical maps in functional neuroimaging using the false discovery rate. Neuroimage 15:870-878.

Greicius MD, Srivastava G, Reiss AL, Menon V (2004) Default-mode network activity distinguishes Alzheimer's disease from healthy aging: evidence from functional MRI. Proc Natl Acad Sci U S A 101:4637-4642.

Gusnard DA, Raichle ME (2001) Searching for a baseline: functional imaging and the resting human brain. Nat Rev Neurosci 2:685-694.

Hayden BY, Smith DV, Platt ML (2009) Electrophysiological correlates of default-mode processing in macaque posterior cingulate cortex. Proc Natl Acad Sci U S A 106:5948-5953.

He BJ, Snyder AZ, Zempel JM, Smyth MD, Raichle ME (2008) Electrophysiological correlates of the brain's intrinsic large-scale functional architecture. Proc Natl Acad Sci U S A 105:16039-16044.

Jensen O, Colgin LL (2007) Cross-frequency coupling between neuronal oscillations. Trends Cogn Sci 11:267-269.

Jerbi K, Ossandón T, Hamamé CM, Senova S, Dalal SS, Jung J, Minotti L, Bertrand O, Berthoz A, Kahane P, Lachaux JP (2009) Task-related gamma-band dynamics from an intracerebral perspective: review and implications for surface EEG and MEG. Hum Brain Mapp 30:1758-1771.

Jerbi K, Vidal JR, Ossandon T, Dalal SS, Jung J, Hoffmann D, Minotti L, Bertrand O, Kahane P, Lachaux JP (2010) Exploring the electrophysiological correlates of the default-mode network with intracerebral EEG. Front Syst Neurosci 4:27.

Kahane P, Landre E, Minotti L, Francione S, Ryvlin P (2006) The Bancaud and Talairach view on the epileptogenic zone: a working hypothesis. Epileptic Disord 8 [Suppl 2]:S16-S26.

Lachaux JP, Rudrauf D, Kahane P (2003) Intracranial EEG and human brain mapping. J Physiol Paris 97:613-628.

Lachaux JP, Fonlupt P, Kahane P, Minotti L, Hoffmann D, Bertrand O, Baciu M (2007) Relationship between task-related gamma oscillations and BOLD signal: New digits from combined fMRI and intracranial EEG. Hum Brain Mapp 28:1368-1375.

Lachaux JP, Jung J, Mainy N, Dreher JC, Bertrand O, Baciu M, Minotti L, Hoffmann D, Kahane P (2008) Silence is golden: transient neural deactivation in the prefrontal cortex during attentive reading. Cereb Cortex $18: 443-450$.

Lakatos P, Karmos G, Mehta AD, Ulbert I, Schroeder CE (2008) Entrainment of neuronal oscillations as a mechanism of attentional selection. Science 320:110-113.

Laufs H, Krakow K, Sterzer P, Eger E, Beyerle A, Salek-Haddadi A, Kleinschmidt A (2003) Electroencephalographic signatures of attentional and cognitive default modes in spontaneous brain activity fluctuations at rest. Proc Natl Acad Sci U S A 100:11053-11058

Laufs H, Hamandi K, Salek-Haddadi A, Kleinschmidt AK, Duncan JS, Lemieux L (2007) Temporal lobe interictal epileptic discharges affect cerebral activity in "Default mode" brain regions. Hum Brain Mapp 28:1023-1032.

Le Van Quyen M, Foucher J, Lachaux J, Rodriguez E, Lutz A, Martinerie J, Varela FJ (2001) Comparison of Hilbert transform and wavelet methods for the analysis of neuronal synchrony. J Neurosci Methods 111:83-98.

Logothetis NK, Pauls J, Augath M, Trinath T, Oeltermann A (2001) Neurophysiological investigation of the basis of the fMRI signal. Nature 412:150-157.

Luo L, Rodriguez E, Jerbi K, Lachaux JP, Martinerie J, Corbetta M, Shulman GL, Piomelli D, Turrigiano GG, Nelson SB, Joëls M, de Kloet ER, Holsboer F, Amodio DM, Frith CD, Block ML, Zecca L, Hong JS, Dantzer R, Kelley KW, Craig AD (2010) Ten years of Nature Reviews Neuroscience: insights from the highly cited. Nat Rev Neurosci 11:718-726.

Manning JR, Jacobs J, Fried I, Kahana MJ (2009) Broadband shifts in local field potential power spectra are correlated with single-neuron spiking in humans. J Neurosci 29:13613-13620.

Mantini D, Perrucci MG, Del Gratta C, Romani GL, Corbetta M (2007) Electrophysiological signatures of resting state networks in the human brain. Proc Natl Acad Sci U S A 104:13170-13175.

Mason MF, Norton MI, Van Horn JD, Wegner DM, Grafton ST, Macrae CN (2007) Wandering minds: the default network and stimulus-independent thought. Science 315:393-395.

Miller KJ, Weaver KE, Ojemann JG (2009) Direct electrophysiological measurement of human default network areas. Proc Natl Acad Sci U S A 106:12174-12177.

Mukamel R, Gelbard H, Arieli A, Hasson U, Fried I, Malach R (2005) Coupling between neuronal firing, field potentials, and FMRI in human auditory cortex. Science 309:951-954.

Niessing J, Ebisch B, Schmidt KE, Niessing M, Singer W, Galuske RA (2005) Hemodynamic signals correlate tightly with synchronized gamma oscillations. Science 309:948-951.

Nir Y, Fisch L, Mukamel R, Gelbard-Sagiv H, Arieli A, Fried I, Malach R (2007) Coupling between neuronal firing rate, gamma LFP, and BOLD fMRI is related to interneuronal correlations. Curr Biol 17:1275-1285.

Pasley BN, Inglis BA, Freeman RD (2007) Analysis of oxygen metabolism 
implies a neural origin for the negative BOLD response in human visual cortex. Neuroimage 36:269-276.

Raichle ME (2009) A paradigm shift in functional brain imaging. J Neurosci 29:12729-12734.

Raichle ME, Mintun MA (2006) Brain work and brain imaging. Annu Rev Neurosci 29:449-476.

Raichle ME, MacLeod AM, Snyder AZ, Powers WJ, Gusnard DA, Shulman GL (2001) A default mode of brain function. Proc Natl Acad Sci U S A 98:676-682.

Ray S, Maunsell JH (2011) Different origins of gamma rhythm and highgamma activity in macaque visual cortex. PLoS biology 9:e1000610.

Ray S, Crone NE, Niebur E, Franaszczuk PJ, Hsiao SS (2008) Neural correlates of high-gamma oscillations $(60-200 \mathrm{~Hz})$ in macaque local field potentials and their potential implications in electrocorticography. J Neurosci 28:11526-11536.

Sakurai K, Takeda Y, Tanaka N, Kurita T, Shiraishi H, Takeuchi F, Nakane S, Sueda K, Koyama T (2010) Generalized spike-wave discharges involve a default mode network in patients with juvenile absence epilepsy: a MEG study. Epilepsy Res 89:176-184.

Scheeringa R, Bastiaansen MC, Petersson KM, Oostenveld R, Norris DG, Hagoort P (2008) Frontal theta EEG activity correlates negatively with the default mode network in resting state. Int J Psychophysiol 67:242-251.

Shmuel A, Augath M, Oeltermann A, Logothetis NK (2006) Negative functional MRI response correlates with decreases in neuronal activity in monkey visual area V1. Nat Neurosci 9:569-577.

Shulman GL, Fiez JA, Corbetta M, Buckner RL, Miezin FM, Raichle ME,
Petersen SE (1997) Common blood flow changes across visual tasks. II. Decreases in cerebral cortex. J Cogn Neurosci 9:648-663.

Singh KD, Fawcett IP (2008) Transient and linearly graded deactivation of the human default-mode network by a visual detection task. Neuroimage 41:100-112.

Spreng RN, Stevens WD, Chamberlain JP, Gilmore AW, Schacter DL (2010) Default network activity, coupled with the frontoparietal control network, supports goal-directed cognition. Neuroimage 53:303-317.

Talairach J, Tounoux P (1993) Referentially oriented cerebral MRI anatomy: an atlas of stereotaxic anatomical correlations for gray and white matter. New York: Thieme.

Tallon-Baudry C, Bertrand O, Delpuech C, Permier J (1997) Oscillatory gamma-band $(30-70 \mathrm{~Hz})$ activity induced by a visual search task in humans. J Neurosci 17:722-734.

Treisman AM, Gelade G (1980) Feature-integration theory of attention. Cogn Psychol 12:97-136.

Uhlhaas PJ, Singer W (2010) Abnormal neural oscillations and synchrony in schizophrenia. Nat Rev Neurosci 11:100-113.

Varela F, Lachaux JP, Rodriguez E, Martinerie J (2001) The brainweb: phase synchronization and large-scale integration. Nat Rev Neurosci 2:229-239.

Weissman DH, Roberts KC, Visscher KM, Woldorff MG (2006) The neural bases of momentary lapses in attention. Nat Neurosci 9:971-978.

Zhang Z, Lu G, Zhong Y, Tan Q, Liao W, Wang Z, Wang Z, Li K, Chen H, Liu Y (2010) Altered spontaneous neuronal activity of the default-mode network in mesial temporal lobe epilepsy. Brain Res 1323:152-160. 